\title{
Instrumentos de evaluación utilizados para la detección de estudiantes con dificultades del aprendizaje en la lecto - escritura de la Educación Escolar Básica del Colegio San Vicente de Paúl de San Ignacio Misiones
}

\author{
Ramona Edith Giménez de Bedoya. \\ edithgimenez40@ hotmail.com \\ Facultad de Humanidades y Ciencias de la Educacion \\ Universidad Nacional de Pilar \\ San Ignacio Misiones, Paraguay
}

\section{RESUMEN}

Esta investigación se llevó a cabo con los alumnos del $3^{\circ}$ Ciclo de la Educación Escolar Básica del Colegio "San Vicente de Paúl" en el periodo que abarca desde el mes de marzo a noviembre del año 2019 y que tiene como fin conocer los instrumentos o test que utilizan los docentes para detectar el tipo de dificultades en la lecto - escritura que tienen los educandos y les impide un aprendizaje significativo. Queriendo detectar cuales son los instrumentos que más utilizan los docentes para evaluar a los alumnos con dificultades de aprendizaje en la lecto - escritura, en base a esto surgen otras interrogantes que como docente necesito comprender el nivel del apoyo profesional que reciben los docentes de la Institución para atender las necesidades educativas de los alumnos con dificultades de aprendizaje en la lectura y la escritura por parte de las autoridades y técnicos de la Institución. Para complementar el resultado del trabajo además se investigó sobre los programas que el MEC está promoviendo para la formación permanente de los docentes y así desarrollar una enseñanza significativa de todos los alumnos. Al realizar lass encuestas y entrevistas se puede observar el proceso de enseñanza - aprendizaje en el aula por parte de los docentes de la Educación Escolar Básica del 3er Ciclo y que favorece a los alumnos, atendiendo a sus necesidades diferenciadas. El tipo de investigación utilizada fue la cuali - cuantitativa que permite enriquecer la recolección de datos. Por lo cual se realizaron Encuestas a 25 Docentes y 50 Alumnos; las Entrevistas a 3 Directivos, y a 5 del Equipo Técnico. Las conclusiones generales a la que se llega es que los docentes de esta Institución cuentan con 
profesionales; Psicólogo y Orientadora que los ayudan a utilizar algunos Test para la detección de problemas de Aprendizaje y en un 36\% utiliza la Batería de Evaluación de los Procesos de escritura PROESC, el 28\% que la Batería de procesos lectores PROLEC - R, un 20\% que la Escala de Magallanes y un $16 \%$ que el Test de análisis de lectura y escritura TALE. En cuanto a los programas que hoy se están incentivando por parte del M.E.C un total de $48 \%$ de los docentes respondieron que es la Educación Inclusiva, un $36 \%$ que son las constantes capacitaciones pedagógicas y un $16 \%$ que es la referente a la capacitación para el mejoramiento de los aprendizajes de niños, jóvenes y adultos. Los docentes encuestados en un $80 \%$ que todas las respuestas son correctas referente al proceso de enseñanza - aprendizaje en el aula que favorece a los alumnos, atendiendo a sus necesidades diferenciadas, un $8 \%$ que las adaptaciones curriculares, otro $8 \%$ que el refuerzo pedagógico y un escaso $4 \%$ que los tutoriales individualizadas y orientadoras.

Palabras claves: Aprendizaje, dificultades, lecto - escritura, alumnos, docentes.

Artículo recibido: 05 de Mayo 2021 Aceptado para publicación: 20 de Junio 2021 Correspondencia: edithgimenez40@ hotmail.com Conflictos de Interés: Ninguna que declarar 


\section{Assessment instruments used for the detection of students with learning difficulties in reading - writing of Basic School Education of the San Vicente de Paúl School in San Ignacio Misiones}

\section{ABSTRACT}

This research was carried out with the students of the 3rd Cycle of Basic School Education of the "San Vicente de Paúl" School in the period from March to November 2019 to demonstrate with what instruments teachers detect the type of difficulties in reading - writing that student have and prevents them from meaningful learning. Its General Objective is to determine the instruments used by teachers to evaluate pupils with learning difficulties in reading - writing. From the statement of the problem the following Specific Objectives arise: To determine the proffessional support that teacher of the Institution receive to meet the educational needs of students with learning difficulties in reading and writing. To complement the result of the work, it was also proposed to Identify the MEC programs that they are promoting for the permanent training of teachers for the meaningful teaching of all students and describe the teaching - learning process in the classroom that favors students, attending to your differentiated needs. Type research: work is done between qualitative and quantitative, wich allows enriching data collection. Study Population: For the Research, Surveys were conducted with 25 teachers and 50 Students, Interviews with 3 Directors, 5 of the Technical Team. The general conclusions: The teachers of this Institution have professionals; Psychologist and Counselor who help them use some tests for the detection of Learning problems and 36\% than the PROESC Writing Process Assessment Battery, 28\% than the PROLEC -R Reading Process Battery, 20\% that the Magallanes scale and 16\% than the TALE Reading and writing analisis test. Regarding the programs that are being encouraged today by the MEC, a total of $48 \%$ of the teachers responded that it is Inclusive Education, $36 \%$ wich are constant pedagogical training and $16 \%$ which refers to training for improving the learning of children, youth and adults. The teachers surveyed $80 \%$ that all the answers are correct regarding the teaching - learning process in the classroom that favors students, attending to their differentiated needs, $8 \%$ that the curricular adaptations, another $8 \%$ that the pedagogical reinforcement and a scarce $4 \%$ than the individualized and orienting tutorials.

Keywords: Learning, difficulties, reading - writing, students, teachers. 


\section{INTRODUCCIÓN}

La historia sobre el estudio e investigación de las dificultades de aprendizaje cuenta con más de dos siglos de historia. Sus orígenes se remontan a principios del siglo XIX, y se extiende en el tiempo hasta el momento actual. Se han realizado varias revisiones bibliográficas en torno a la cuestión de las Dificultades del Aprendizaje (DA) (Vila, 2013).

En España hoy en día se consideran cuatro fases fundamentales en la historia de las DA, de las cuales las tres primeras fueron propuestas por Wiederholt en 1974 y la cuarta por Lerme en 1.989 (Vila \& Gutiérrez., 2013). Estas fases son: 1. Fase de Fundación, que iría desde el origen del estudio de las DA en 1800 hasta 1940. 2. Fase de Transición (1940 a 1963) 3. Fase de Integración (1963 a 1980) 4. Fase de consolidación (1980 hasta la actualidad)

En la actualidad, Zubiarre, y Rodríguez, (1979):

Existen suficientes evidencias que demuestran que las necesidades educativas especiales surgen de la interacción de múltiples factores, algunos inherentes al propio niño (como por ejemplo las discapacidades), otros asociados a sus circunstancias socioambientales y culturales; sin olvidar, aquellos relacionados con la respuesta, y oportunidades educativas que se les ofrece. Desde esta perspectiva, se considera que las necesidades educativas especiales tienen un carácter interactivo, es decir, dependen de las condiciones del propio alumno y las características del contexto educativo en el cual se manifiestan. (pp.153-168)

El carácter interactivo y contextual de las necesidades educativas especiales, provoca ambigüedad, pues un niño/a puede ser considerado, o no, con dichas necesidades dependiendo del contexto. Para resolver esta inconsistencia, se introdujo la denominación barrera para el aprendizaje y la participación. En el caso españolconsiderando las peculiaridades del concepto de necesidades educativas especiales-, y sobre todo el carácter "transitorio", frente a las dificultades de aprendizaje, que tendrían la consideración de "permanentes". (Wolf, 2005)

En Uruguay Igualmente, en la materia troncal de los Planes de Estudios de Magisterio denominada "Bases Psicopedagógicas de la Educación Especial", se recoge la 
recomendación del Informe Warnock de incluir un mínimo de formación en Educación Especial para todos los profesores y no sólo para los especialistas. De dicho informe se desgajó una visión de las características que definían las necesidades educativas especiales: - El carácter gradual y la posible dilatación de las DA. - El carácter relativo del concepto (cambiante en base al contexto). - La demanda de respuestas pedagógicas específicas en cada etapa de la escolaridad. - La necesidad de recursos extraordinarios para reforzar sus aprendizajes.

La preocupación por el rendimiento escolar de los niños y en particular por conocer sobre los trastornos en lectura que presentaban algunos alumnos para los cuales se hacía necesario elaborar programas adecuados de intervención, llevó a que en 1962 se publicara en la revista "Archivos de Pediatría del Uruguay" un trabajo llamado "La dislexia de evolución: estudio de 35 casos personales” de Bauzá y colaboradores en 1.962. Louzán (1965) destacó que hay una pluralidad de síntomas que llevarían a diagnosticar la dislexia: la discordancia entre el Cedula de Identidad y el nivel lector alcanzado en lectura y escritura; nivel de lectura y escritura por debajo del promedio de la clase; diferencias con el rendimiento alcanzado en otras áreas; errores en lectura del tipo de rotaciones, inversiones, confusiones y sustituciones de letras; dificultades de orden simbólico; alteraciones en el lenguaje; dificultades de percepción, de esquema corporal, de motricidad, de memoria, en la capacidad de abstracción y generalización y desorientación espacial; trastornos neurológicos o emocionales; y antecedentes familiares. Criterios similares aparecen en los trabajos de Tuana $(1965,1967)$

A partir de las publicaciones mencionadas, la mayor parte de los trabajos presentados hasta la fecha han vinculado la dislexia de evolución con diversos factores, tales como los trastornos en el habla, los problemas de lateralidad, y los déficits perceptivos y motrices, así como por factores emocionales .También se asocia con problemas en la producción escrita como la disortografía (Carbonell de Grompone, 1975) o dificultades a nivel morfosintáctico (Zubiarre y Rodríguez, 1979)

En Argentina Signorini y Borzone de Manrique (2003) profundizaron el empleo de las estrategias ortográficas y fonológicas en alumnos de segundo año básico y su eventual éxito en el aprendizaje. Los resultados mostraron "un efecto de complejidad ortográfica cuando la variable dependiente fue el tiempo de lectura" Sus resultados también confirman el peso que tienen las estrategias fonológicas, especialmente cuando los niños 
están aprendiendo a escribir. Ese mismo año, Diuk, Signorini y Borzone (2003a) investigaron el aprendizaje lector en niños de nivel socioeconómico bajo, de tercer año, comparándolos con niños de NSE medio de primer año. Sus resultados mostraron que los niños de nivel bajo presentan un patrón evolutivo cualitativamente similar al de los niños de nivel medio, aunque con retraso en su desarrollo. También encontraron que "los niños malos lectores compensan sus limitaciones de procesamiento fonológico apoyándose en su conocimiento ortográfico”.

En Perú 1994 los psicólogos Torres y Luna reunieron diversas experiencias efectuadas con niños que presentaban dificultades de aprendizaje en un libro titulado "Problemas de Aprendizaje". Presentan resultados de programas correctivos, sistemas motivacionales y estrategias de intervención para mejorar habilidades escolares, como aritmética, ortografía y lectura. (Alarcón, 2000).

En Paraguay la psicología también ha experimentado progresos en el estudio de las Dificultades de Aprendizaje, pero sus niveles de visibilidad todavía son escasos. En 2007 apareció en Asunción un libro colectivo publicado por la Asociación Paraguaya de Neuropsicología, que aborda algunos temas relacionados con el aprendizaje de la lectura y de las matemáticas. Estos capítulos son resultado de investigaciones que siguen el modelo Fisiológico y Fisiopatológico del profesor argentino Juan E. Azcoaga, quien ha orientado al grupo de investigadores paraguayos

Una cuestión que se plantea constante preocupación en los diferentes profesionales que intervienen en el sistema educativo, en las aulas se ha observado la influencia negativa de las dificultades de aprendizaje en la adquisición de distintas habilidades básicas y la problemática a nivel académico que surge relacionada con la adquisición de la lectoescritura y las habilidades matemáticas.

Estas dificultades suponen para los alumnos una condición que interfiere en su vida escolar, al favorecer una discrepancia significativa entre su verdadero potencial y su rendimiento académico, repercutiendo, consecuentemente, tanto en su autoestima como en su relación con los compañeros y con el aprendizaje (Miranda et al, 2002).

Una estrategia para superar esta dificultad fue efectuar una investigación comparativa entre niños con buen y bajo rendimiento escolar, pertenecientes a las mismas escuelas y aulas, equiparadas por edad, Cedula de Identidad, sexo y Niveles Socio Económicos (NSE) durante cuatro años. (Azcoaga, 1979) El seguimiento mostró que las diferencias 
en el aprendizaje entre alumnos de bajo NSE no se explicaban por diferencias en los factores socio económico. Las mayores diferencias en el aprendizaje estuvieron en las pruebas de conciencia fonológica, memoria de secuencia de letras que formaran una palabra, comprensión auditiva de una historia, y en abstracción verbal.

Esos resultados mostraron que las diferencias socioeconómicas no aparecían ser la principal causa de las diferencias en el rendimiento entre los buenos y los malos lectores del grupo estudiado (Bravo, 1995). El resultado de este seguimiento de cuatro años puede asociarse con otro estudio de seguimiento entre primero y cuarto año básico de 227 alumnos recién ingresados al primer año. El estudio consistió en determinar cuáles eran las principales variables predictivas del rendimiento en lectura a fines del cuarto año, comparando también los buenos y malos lectores.

Todos los sujetos pertenecían a un nivel socioeconómico bajo. Los resultados de la matriz de correlaciones entre las pruebas iniciales y la lectura final y de regresión s sobre la lectura, mostraron que las variables con mayor predictividad del lenguaje escrito fueron el conocimiento inicial de las letras, el reconocimiento de algunas palabras escritas y la conciencia fonológica, todos ellos evaluados al momento de ingresar a primer año (Bravo, 2006). Las diferencias en lectura entre los niños con buen y mal rendimiento inicial al ingresar al primer año se mantuvieron hasta fines de cuarto año y no se explicaron por sus diferencias socio económicas. En la actualidad la sociedad se apoya en la escuela como institución formal que hace posible el proceso educativo.

La escuela es un medio educativo puesto por y en la sociedad a disposición del niño, de la familia y de la sociedad. El maestro efectúa su mediación pedagógica en una ósmosis de vida y de trabajo con alumnos, familias y miembros de la comunidad (García, 2001). En este sentido, Suárez (2005, p 65) expresa que el docente se convierte en" Fomentador de análisis, inductor de cambios, activador de búsqueda, motivador y facilitador de experiencias, suscitador de discusión y crítica, generador de hipótesis, planeador de problemas y alternativas, promotor y dinamizador de cultura, frente a un grupo estudiantil que piensa, crea, transforma, organiza y estructura conocimientos en un sistema personal y dinámico".

Actualmente se dan numerosos estudios e investigaciones sobre la importancia de detectar las Dificultades de Aprendizaje para tomar acciones que redunden en beneficio 
del aprendizaje del educando. (Jiménez et al, 2009), (Cuetos, 2009). Lo cual es el objetivo primordial que me mueve a realizar esta investigación, a pesar que actualmente los docentes tienen el apoyo significativo desde el propio Ministerio de Educación y Ciencias que habilitan cursos de capacitación constante de los mismos especialmente los que se inician en la labor educativa. En el caso del Colegio elegido para esta investigación tiene diversos profesionales técnicos que apoyan a los docentes cotidianamente en las diversas situaciones.

\section{MATERIAL Y MÉTODO}

Descriptiva: porque se pretendió dar a conocer los instrumentos utilizados por los docentes en el proceso de detección de los alumnos con problemas de aprendizajes en la E.E.B del $3^{\circ}$ Ciclo, para cotejar con las situaciones observadas al desarrollar los procesos de clases y así hacer las adecuaciones necesarias para el mejoramiento en todas las disciplinas de los educandos. Cualitativo: porque se estableció la descripción y el análisis de los registros, por medio de técnica de las entrevistas a fin de brindar un estudio certero. Cuantitativo porque se detectó los diversos casos de problemas de aprendizaje en la lecto - escritura en este caso el Test de PROLEC, que fueron analizados por medio de encuestas cuantificadas para su representación gráfica.

El universo del trabajo que se realizó: encuesta dirigida a 25 Docentes del $3^{\circ}$ ciclo de la EEB con cuestionarios sobre la labor docente y el apoyo que reciben como funcionarios del Ministerio de Educación y Ciencias de elaboración propia, Batería PROLEC por ser un Test conocido. 50 alumnos del $3^{\circ}$ ciclo de la EEB, 3 Directivos del Colegio, 5 Equipo Técnico, 5 Técnicos de la Supervisión Pedagógica del M.E.C. La muestra está constituida por el $70 \%$ de la población, que consiste en 18 Docentes del $3^{\circ}$ ciclo de la EEB, 35 Alumnos del $3^{\circ}$ ciclo de la EEB, 2 Directivos del Colegio, 4 Equipo Técnico, 4 Técnicos de la Supervisión Pedagógica del M.E.C

Se utilizó la entrevista y la encuesta como técnicas de la recolección de datos. El primero, consiste en una Encuesta de elaboración propia con opciones directas dirigidas a docentes y directivos de la Institución. El otro único instrumento que se utilizó para registrar los procesos de lectura y escritura del grupo de estudiantes del Colegio "San Vicente de Paúl"“ consistente a la Batería PROLEC ${ }^{1}$ que proporciona información no sólo de las dificultades que presenta el niño frente a la lectura, sino cuáles son los

\footnotetext{
${ }^{1}$ Este Instrumento esta revisado y aprobado, que tiene como Autores a Fernando Cuetos, Blanca Rodríguez, Elvira Ruano y David Arribas.
} 
procesos cognitivos responsables de esas dificultades, además de proporcionar algunas herramientas educativas para la recuperación de los procesos que presenten dificultades. La batería está compuesta por nueve pruebas que tratan de explorar los principales procesos lectores, desde los más básicos a los más complejos. Consiste en Las subcategorías que se tuvieron en cuenta para el área de escritura fueron: a) omisión y cambio de letras, b) segmentación en la escritura, c) estructura de la oración, d) Intención comunicativa, e) uso de mayúsculas, puntuación, ortografía y f) producción textual. Para el caso de la lectura, se tuvieron en cuenta la comprensión y el ritmo al leer por parte de los estudiantes.

\section{RESULTADOS}

\section{1- Los test de evaluación a alumnos con problemas de Aprendizajes son:}

Los docentes de esta Institución gracias a contar con profesionales Psicólogo y Orientadora son adiestrados a utilizar algunos Test para la detección de problemas de Aprendizaje que en la actualidad existen varios pero para esta investigación se centra solo en algunos según la preferencia de los docentes que respondieron en un $36 \%$ que la Batería de Evaluación de los Procesos de escritura PROESC, el 28\% que la Batería de procesos lectores PROLEC - R, un 20\% que la Escala de Magallanes y un $16 \%$ que el Test de análisis de lectura y escritura TALE.

Gráfico 1: Test para la detección de problemas de aprendizaje

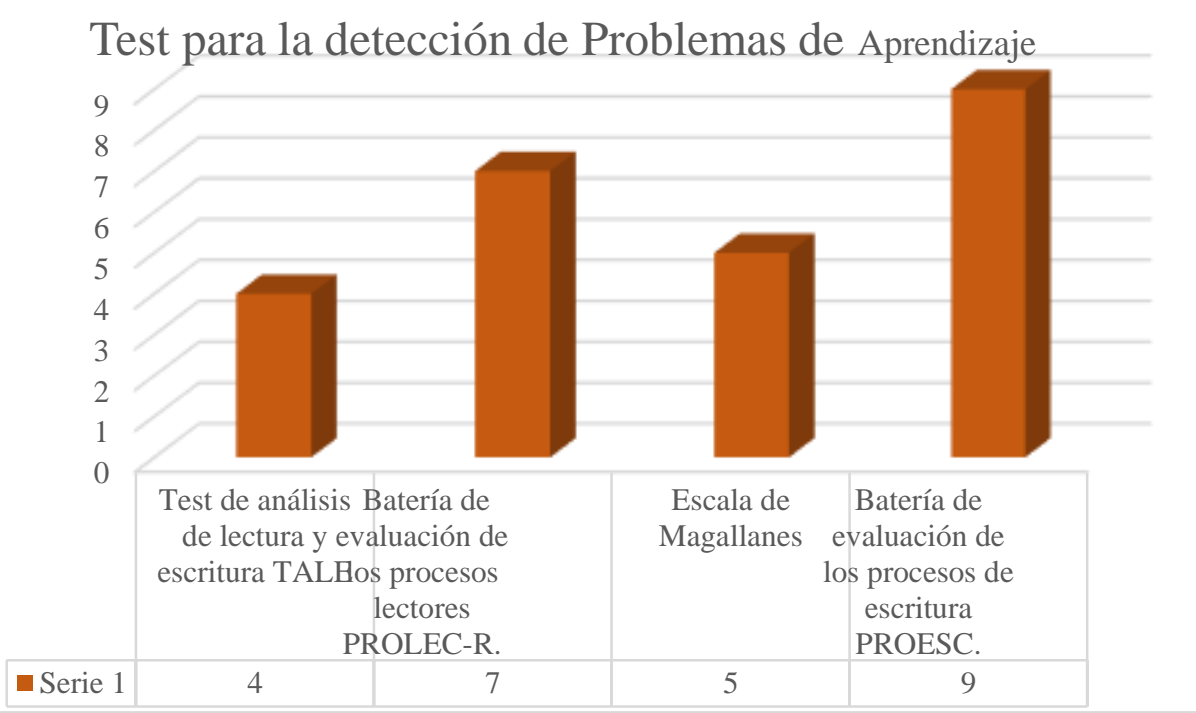

Fuente: Elaboración propia. Datos obtenidos a través del trabajo de campo realizado en el Colegio "San Vicente de Paúl" de San Ignacio Misiones. 
- Identificar los programas del MEC que están promoviendo para la formación permanente de los docentes para la enseñanza significativa de todos los alumnos.

En cuanto a los programas que hoy se están incentivando por parte del M.E.C un total de $48 \%$ de los docentes respondieron que es la Educación Inclusiva, un 36\% que son las constantes capacitaciones pedagógicas y un $16 \%$ que es la referente a la capacitación para el mejoramiento de los aprendizajes de niños, jóvenes y adultos. Estos son programas que ayudan en la preparación de los docentes para mejorar su labor educativa y gracias a eso la comunidad educativa se siente acompañada.

Gráfico 2: Programas implementados por El MEC

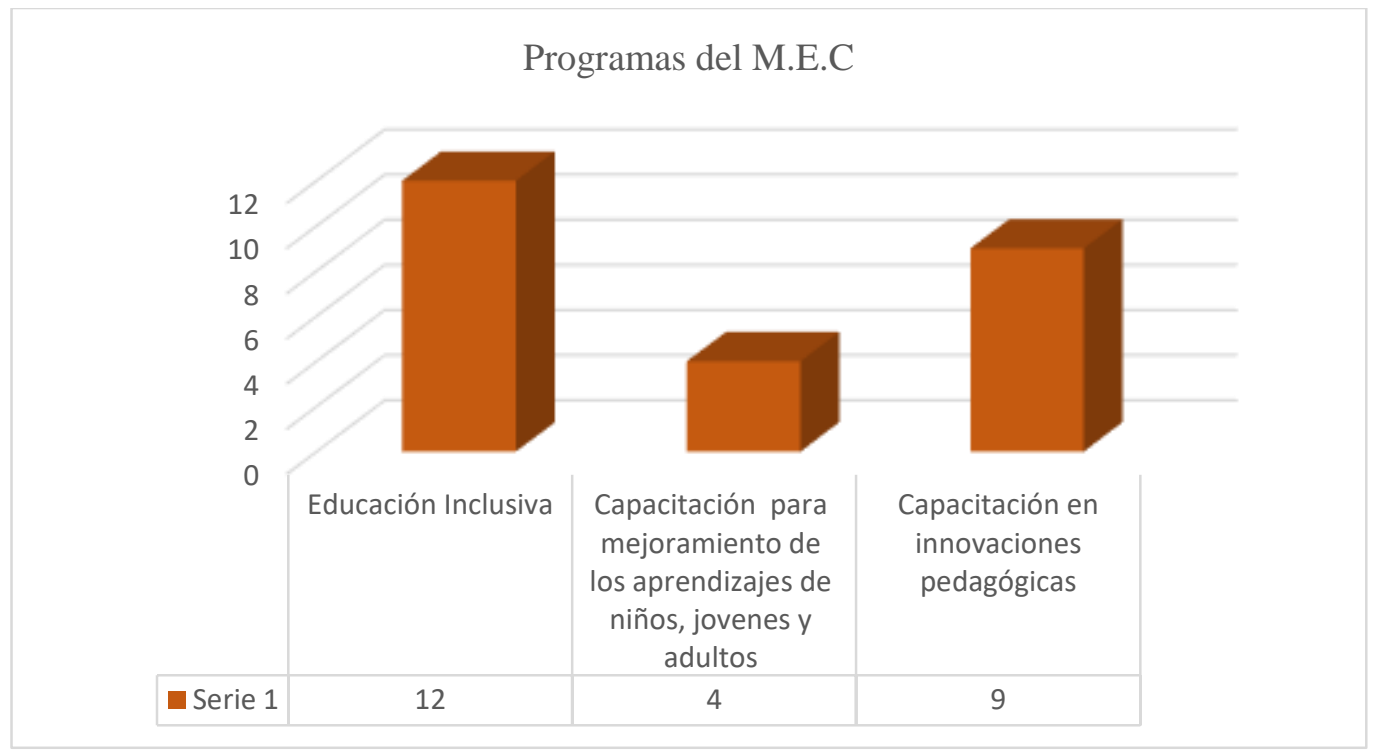

Fuente: Elaboración propia. Datos obtenidos a través del trabajo de campo realizado en el Colegio "San Vicente de Paúl" de San Ignacio Misiones.

- Determinar el apoyo profesional que reciben los docentes de la Institución para atender las necesidades educativas de los alumnos con dificultades de aprendizaje en la lectura y la escritura.

Los docentes encuestados respondieron en un $100 \%$ que tienen apoyo de personal especializado sea Psicóloga, Orientadora, que le proporciona asesoramiento en la aplicación de los Test de Aprendizaje, pues muy contadas instituciones cuentan con un equipo multidisciplinario en beneficio de los educandos. Este acompañamiento de Profesionales ayuda a los docentes ya sea con pocos o muchos años de experiencia pues cada vez hay más factores que influyen en la función educativa por lo tanto se necesita de la ayuda de todos los actores. 
Gráfico 3: Personal De Apoyo

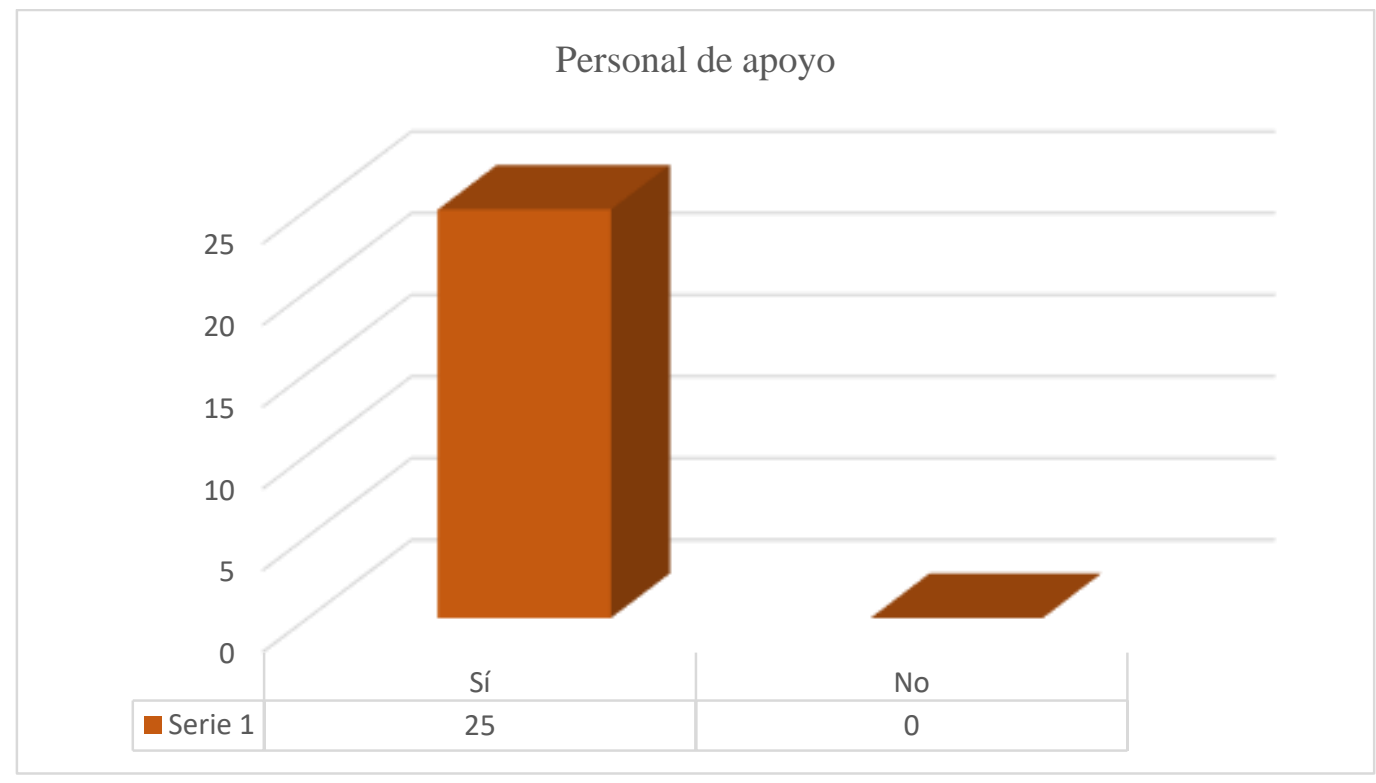

Fuente: Elaboración propia. Datos obtenidos a través del trabajo de campo realizado en el Colegio "San Vicente de Paúl" de San Ignacio Misiones.

- Describir el proceso de enseñanza - aprendizaje en el aula que favorece a los alumnos, atendiendo a sus necesidades diferenciadas.

Los docentes encuestados en un $80 \%$ respondieron que el conglomerado del refuerzo pedagógico, las adaptaciones curriculares, los tutoriales individualizados y orientados favorecen al proceso de enseñanza - aprendizaje en el aula, atendiendo a las necesidades diferenciadas de los alumnos; mientras que un escaso $8 \%$ respondió que son importantes las adaptaciones curriculares, otro $8 \%$ que el refuerzo pedagógico y un mínimo 4\% que los tutoriales individualizados y orientados. En las entrevistas realizadas a directivos y funcionarios de la Supervisión en su mayoría coincidieron que actualmente se tienen programas y adecuaciones en la formación de los alumnos docentes a fin de enfrentar su labor con herramientas necesarias para incentivar el aprendizaje significativo de los alumnos aun con dificultades de aprendizaje.

La coherencia de las estrategias didácticas y la actitud del profesorado respecto a la diversidad de ideas, de experiencias, de actitudes, de estilos de aprendizaje, de ritmos, de capacidades, de intereses; esto en palabras de Giné, 2001 marcará de manera muy relevante, las características del ambiente de aprendizaje que se dé en el aula, en aras de brindar una atención a la diversidad. 
Gráfico 4: Procesos de Enseñanza

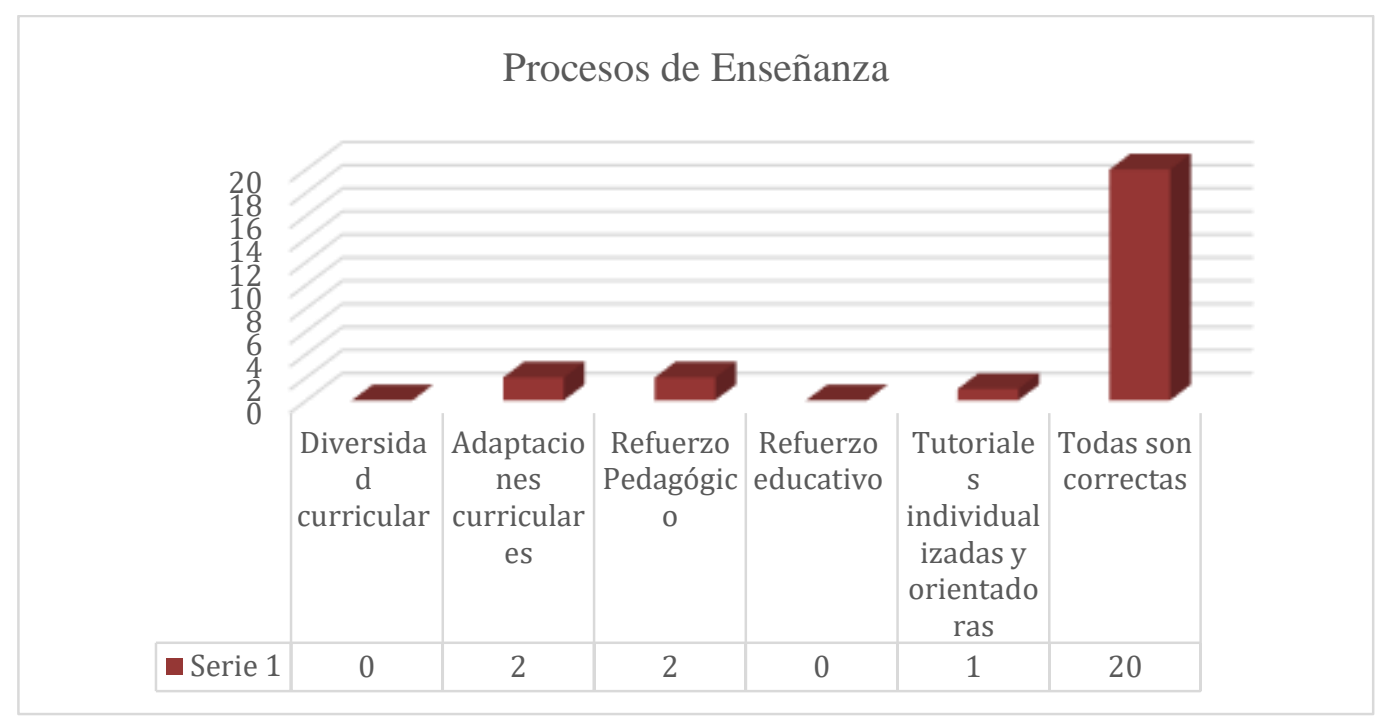

Fuente: Elaboración propia. Datos obtenidos a través del trabajo de campo realizado en el Colegio "San Vicente de Paúl" de San Ignacio Misiones.

\section{DISCUSIÓN}

- Instrumentos utilizados por los docentes para la detección de dificultades del aprendizaje.

Los docentes de esta Institución gracias a contar con profesionales Psicólogo y Orientadora son adiestrados a utilizar algunos Test para la detección de problemas de Aprendizaje entre sus alumnos, pero optando dependiendo del grupo a su cargo en el año lectivo un alto porcentaje utiliza la Batería de Evaluación de los Procesos de escritura PROESC y un menor porcentaje la Batería de procesos lectores PROLEC - R. En la Universidad de Sevilla se realizó un proyecto de intervención con una niña de 7 años, actualmente cursando el $2^{\circ}$ curso de Educación Primaria, recoge toda la información necesaria para llevar a cabo la intervención, relacionada con el concepto de dislexia, sus causas, características, errores típicos, etc., el concepto de competencia en comunicación lingüística, el desarrollo de la lectoescritura en la etapa de Educación Primaria y el uso de la metodología cualitativa a través de diferentes instrumentos, tales como la entrevista semiestructurada y la observación. Para ello ha sido necesario realizar una entrevista al profesional del centro al que acude la alumna y realizar las pruebas Prolec y Proesc para conocer su nivel inicial en lectoescritura. (Solis, 2.018) 


\section{- Formación del Docente en la práctica - Docente - EEB - $3^{\circ}$ Ciclo.}

En cuanto a los programas que hoy se están incentivando por parte del M.E.C la gran mayoría de los docentes respondieron que es la Educación Inclusiva, otros que son las constantes capacitaciones pedagógicas y pequeño grupo respondieron que es lo referente a la capacitación para el mejoramiento de los aprendizajes de niños, jóvenes y adultos.

En una investigación que hemos realizado sobre una encuesta nacional a 807 maestros noveles de Uruguay (Aguerrondo y Vezub, 2003) el 46\% consideró que habían sido insuficientemente tratadas durante su formación las cuestiones pedagógicas y didácticas, y el $69 \%$ opinó que su preparación inicial sobre estrategias para trabajar en contextos socioculturales diversos fue insuficiente. La formación suele abordar situaciones de clase e instituciones "tipo", generalmente escuelas urbanas que atienden a una población más o menos homogénea y con bajo nivel de conflictividad social. No se abordan los métodos de enseñanza en contextos adversos, con población escolar heterogénea, afectada por la pobreza y por graves problemas sociales.

- Profesionales técnicos de la Institución y de la Supervisión Pedagógica del Nivel.

En las entrevistas realizadas a directivos y funcionarios de la Supervisión en su mayoría coincidieron que actualmente se tienen programas y adecuaciones en la formación de los alumnos - docentes a fin de enfrentar su labor con herramientas necesarias para incentivar el aprendizaje significativo de los alumnos aun con dificultades de aprendizaje.

Stainback, Staimback, y Jackson (1999), Arnáiz (1999), Blanco (2000) y Yadarola (2006), promueven que las aulas deben ser utilizadas como espacios sociales donde se propicie un clima de diversidad entre los alumnos, se generen espacios de participación activa, solidaria y organizada; con el propósito de dar respuesta a través de un aprendizaje cooperativo a la heterogeneidad de cada uno de los estudiantes, brindándoles el apoyo que necesiten entre todos.

Referente a la percepción de los docentes en aula de las dificultades que presentan los alumnos en su mayoría corresponde al razonamiento cuantitativo, después se observan las dificultades para el cálculo mental, la dificultad de precisión para leer y la falta de fluidez de comprender lo leído en un menor porcentaje. 
Según informes las Dificultades en el Aprendizaje son equivalentes a las Necesidades Educativas Especiales. Expresión inglesa que procede del Informe Warnock (1987), y que tuvo, por un lado, el objetivo de unificar todas las categorías tradicionales de la Educación Especial; y por otro, suprimir etiquetas de escaso valor nosológico, a veces, y de efectos nocivos sobre los alumnos y el curso de sus problemas. El modelo educativo español vigente, se sitúa en la línea de las Necesidades Educativas Especiales, que se acoge a la Declaración de Salamanca de 1994, que dio lugar al informe sobre necesidades educativas especiales, en el que se aboga por la abolición de las clasificaciones y se acentúa la importancia de analizar las necesidades especiales que presenta cada persona. El informe es el resultado de una pregunta parlamentaria del 26 de abril de 1978 a la que el Gobierno Británico se comprometió a responder.

\section{CONCLUSIÓN}

Aunque las dificultades de aprendizaje siempre van a estar presentes en los procesos de enseñanza-aprendizaje, depende de los docentes que detecten a los alumnos que los poseen para así tratar de mejorar el aprendizaje significativo de los mismos.

Los docentes de esta Institución gracias a contar con profesionales Psicóloga y Orientadora son adiestrados a utilizar algunos Test para detectar problemas de Aprendizaje en los alumnos a sus cargos y así promover el aprendizaje significativo en las aulas. En cuanto a los programas que hoy se están incentivando por parte del M.E.C los docentes valoran el de la Educación Inclusiva, como así también las constantes capacitaciones pedagógicas para el mejoramiento de los aprendizajes de niños, jóvenes y adultos.

Los docentes tienen el apoyo de la comunidad educativa para que los alumnos del $3^{\circ}$ Ciclo tengan un aprendizaje significativo en todas las disciplinas del nivel.

En las entrevistas realizadas a directivos y funcionarios de la Supervisión en su mayoría coincidieron que actualmente se tienen programas y adecuaciones en la formación de los alumnos - docentes a fin de enfrentar su labor con herramientas necesarias

\section{REFERENCIAS BIBLIOGRÁFICAS}

Aguerrondo, I. y Vezub, L. (2003). Los primeros años como maestro. Desarrollo profesional de los docentes uruguayos. Programa de modernización y Formación Docente. Montevideo: ANEP - MEMFOD 
Alarcón Reynaldo (2000). Historia de la Psicología en el Perú. Lima. Universidad Ricardo Palma

Armele, M., Barriocanal L., Ferreira M.E., Ojeda M, Rumich D. y Scavone J. (2007) Neuropsicología, aprendizaje y ciencia. Asunción. Asociación Paraguaya de Neuropsicología.

Azcoaga, JE., Derman, B., Iglesias, P.A. (1979). Alteraciones del aprendizaje Escolar. Diagnóstico, fisiopatología, tratamiento. Buenos Aires: Paidós. Azcoaga J.E (1979) Aprendizaje fisiológico y aprendizaje pedagógico. Buenos Aires: El Ateneo.

Bravo L. (1990) Psicología de las Dificultades del aprendizaje escolar. Santiago de Chile: Editorial Universitaria ( 8 a edición 2009).

Carbonell de Grompone, M. (1975). Colonialismo y dislexia. En El lenguaje lecto escrito y sus problemas. Buenos Aires: Editorial Médica Panamericana.

Cuetos F. 2009 Dislexias evolutivas: un puzzle por resolver. Revista de Logopedia, Foniatría y Audiología. 2009;29:78-84.

Diuk B., Signorini, A., Borzona, A.M. Las estrategias tempranas de lectura de palabras en niños de $1^{\circ}$ a $3^{\circ}$ año de educación general básica: Un estudio comparativo entre niños procedentes de distintos sectores sociales. Psykhé, 12, 51-62.

García, A. (2001). Teorías e Instituciones de la Educación. Una aproximación Sociológica. Madrid, Padilla Libros Editores.

Giné, C. (2001). Inclusión y sistema educativo. Ponencia presentada en el III Congreso La atención a la diversidad en el sistema educativo, Universidad de Salamanca, Salamanca.

Jiménez JE, Rodríguez C, Ramirez G. 2009 Spanish developmental dyslexia: prevalence, cognitive profile, and home literacy experiences. J Exp Child Psychol. ; 103:167-85.

Louzan, M. (1965). Diagnóstico Global del retardo en lectura. Boletín del IIN. 155, 776-784. Montevideo, Uruguay.

Louzan, M. (1965). Lateralidad, Estructuración Espacial y Dislexia. Actas y Trabajos del I Seminario sobre Dislexia en el Uruguay. 110-122. 
Miranda, A., Vidal-Abarca, E. y Soriano, M. (2002). Evaluación e intervención psicoeducativa en dificultades de aprendizaje. Madrid: Pirámide.

Solís, J (2018) Facultad Ciencias de la Educación Grado en Pedagogía Madrid España.

Stainback, S.; Staimback, W.; y Jackson, J. (1999). Hacia las aulas inclusivas. En Stainback, S y Staimback, W. (Cood.) Aulas Inclusivas. Editorial Nancea. Madrid: España.

Suárez, R. (2005). La Educación. Teorías Educativas. Estrategias de Enseñanza Aprendizaje. Madrid, Trilla.

Tuana, E. (1965). Diagnóstico analítico de las dificultades en lectura. Boletín de IIN. Montevideo, $\mathrm{N}^{\mathrm{o}} 155$, pp.785-796.

Tuana, E. (1967). Dificultades en el aprendizaje de la lectura y escritura. Boletín de la Inspección Nacional de Escuelas de Práctica. Montevideo, 11,1 - 12

Vila, J. O., \& Gutiérrez, F. (2013). Manual básico de dificultades de aprendizaje, concepto, evaluación e intervención. UNED: Sanz y Torres

Vygotsky, L.S. 1982-84. Sobranie socinenii [Obras completas], Vols. I-VI. Moscú, Pedagogika.

Warnock, M. (1987): Encuentro sobre Necesidades de Educación Especial. Revista de Educación, $\mathrm{n}^{\circ}$ extraordinario, 45-73.

Wolf, M., \& Denckla, M. B. (2005). RAN/RAS: The rapid automatized naming and rapid alternating stimulus tests. Austin, TX: Pro-ed.

Zubiarre, M. y Rodríguez, L. (1979). La comunicación escrita en el niño disléxico. Revista APPIA. Montevideo. 6, Fascículo 1-2, pp.153-168. 